\title{
Introduction of percutaneous-tunneled transfontanellar external ventricular drainage in the management of hydrocephalus in extremely low-birth-weight infants
}

\author{
Mino Zucchelli, MD, ${ }^{1}$ Mariella Lefosse, MD, ${ }^{1}$ Luigi Corvaglia, MD, ${ }^{2}$ Silvia Martini, MD, ${ }^{2}$ \\ Fabrizio Sandri, MD, ${ }^{3}$ Silvia Soffritti, MD, ${ }^{3}$ Gina Ancora, MD, ${ }^{4}$ Palma Mammoliti, MD, ${ }^{4}$ \\ Giancarlo Gargano, MD, ${ }^{5}$ and Ercole Galassi, MD'
}

\begin{abstract}
${ }^{1}$ Pediatric Neurosurgery, IRCCS Istituto delle Scienze Neurologiche di Bologna, Bellaria Hospital; ${ }^{2}$ Neonatal Intensive Care Unit, Department of Medical and Surgical Sciences, S. Orsola-Malpighi University Hospital; ${ }^{3}$ Neonatal Intensive Care Unit, Maternal and Pediatrics Department, Maggiore Hospital, Bologna; ${ }^{4}$ Neonatal Intensive Care Unit, Infermi Hospital, Rimini; ${ }^{5}$ Neonatal Intensive Care Unit, Obstetrics, Gynecology and Pediatrics Department, Arcispedale Santa Maria Nuova Hospital, IRCCS, Reggio Emilia, Italy
\end{abstract}

OBJECTIVE Hydrocephalus treatment in extremely low-birth-weight (ELBW) infants still represents a challenge for the pediatric neurosurgeon, particularly when the patient weighs far less than $1000 \mathrm{~g}$. In such cases, the benefits in terms of neurological outcome following early treatment do not always outweigh the surgical risks, especially considering the great difference in the surgical risk before patient weight increases. To assess the efficacy and reliability of a percutaneous-tunneled, transfontanellar external ventricular drain (PTTEVD) in ELBW infants, the authors started a new protocol for the early surgical treatment of hydrocephalus.

METHODS Ten cases of posthemorrhagic hydrocephalus (PHH) in ELBW infants ( 5 cases $<700 \mathrm{~g}$, range for all cases 550-1000 g) were treated with a PTTEVD that was implanted at bedside as the first measure in a stepwise approach.

RESULTS The average duration of the procedure was 7 minutes, and there was no blood loss. The drain remained in place for an average of 24 days (range 8-45 days). In all cases early control of the hydrocephalus was achieved. One patient had a single episode of CSF leakage (due to insufficient CSF removal). In another patient Enterococcus in the CSF sample was detected the day after abdominal surgery with ileostomy (infection resolved with intrathecal vancomycin). One patient died of Streptococcus sepsis, a systemic infection existing prior to drain placement that never resolved. One patient had Pseudomonas aeruginosa sepsis prior to drain insertion; a PTTEVD was implanted, the infection resolved, and the hydrocephalus was treated in the same way as with a traditional EVD, while the advantages of a quick, minimally invasive, bedside procedure were maintained. Once a patient reached $1 \mathrm{~kg}$ in weight, when necessary, a ventriculoperitoneal shunt was implanted and the PTTEVD was removed.

CONCLUSIONS The introduction of PTTEVD placement in our standard protocol for the management of PHH has proved to be a wise option for small patients.

http://thejns.org/doi/abs/10.3171/2016.1.PEDS15563

KEY WORDS extremely low-birth-weight infants; hydrocephalus

$\Lambda$ LTHOUGH the improvement of treatment modalities in neonatal intensive care units (NICUs) has increased the survival rates of extremely low-birthweight (ELBW) infants $(<1000 \mathrm{~g})$, severe intraventricular hemorrhage (IVH) and subsequent posthemorrhagic hydrocephalus (PHH) still represent serious complications of premature birth and major causes of neurodevelopmental impairment. ${ }^{11}$ The management of PHH in ELBW infants is complex: Although this population is the most severely affected, at present there is no consensus on the optimal timing or best modality of intervention. The overall incidence of IVH in ELBW infants ranges from $7 \%$ to $23 \%$; $33 \%$ of these infants develop posthemorrhagic ventricular dilation (PVD), and $15 \%{ }^{1}$ of those with PVD require neurosurgical treatment.

Moreover, regardless of patient weight, once the ven-

ABBREVIATIONS ELBW = extremely low birth weight; EVD = external ventricular drain; IVH = intraventricular hemorrhage; $\mathrm{NICU} \mathrm{=} \mathrm{neonatal} \mathrm{intensive} \mathrm{care} \mathrm{unit;} \mathrm{PHH}=$ posthemorrhagic hydrocephalus; PTTEVD = percutaneous-tunneled, transfontanellar EVD; PVD = posthemorrhagic ventricular dilation.

SUBMITTED September 17, 2015. ACCEPTED January 13, 2016.

INCLUDE WHEN CITING Published online March 25, 2016; DOI: 10.3171/2016.1.PEDS15563. 
tricular dilation has become progressive, early treatment of the hydrocephalus may reduce the period of brain injury, with a consequent reasonable improvement in the final global outcome. ${ }^{16}$ Extremely low-birth-weight infants represent a subgroup of hydrocephalic patients in whom early surgical treatment of the hydrocephalus represents a challenge for many reasons: thinness of skin, risk of skin ulceration, CSF fistula, infection, and pulmonary hemodynamic instability together with the inability to move the infant out of the incubator. Concern about these frequent complications ${ }^{12}$ of standard treatments can delay surgery, and the infant's weight increase in this period of delay will reduce the operative risk. Indeed, from a surgical point of view, operating on an infant weighing $950 \mathrm{~g}$ is incomparably easier and safer than performing the same operation on the same patient when he or she weighs $500 \mathrm{~g}$ (both cases are considered ELBW) since the lower the weight, the fewer the feasible surgical options and the higher the risks.

A possible final treatment for hydrocephalus in children is the implantation of a permanent CSF shunt. ${ }^{7}$ In ELBW infants, such invasive surgery is impractical because of the aforementioned risks and the presence of blood and clots within the ventricles, which must be removed before shunt implantation. To achieve the goal of implantation, several standardized techniques have been proposed over time: repeated transfontanellar ventricular puncture and intermittent lumbar puncture, standard external ventricular drainage, Ommaya reservoir implantation, and ventriculosubgaleal shunt implantation. None of these procedures can be considered without risks even in full-term babies, and operating on such small patients would increase the probability of serious complications.

We introduced and modified in our treatment protocol for PHH an already described technique ${ }^{9}$ usually reserved for infants under $1000 \mathrm{~g}$, which helps to achieve early control of the hydrocephalus and to gain time while waiting for the weight and general condition of these small patients to allow safer surgical implantation of a permanent shunt.

\section{Methods}

In the last 3 years (first case in December 2012, last case in May 2015), we have treated 10 cases of PHH in ELBW infants ( 5 cases $<700 \mathrm{~g}$, range for all cases 550-1000 g) by implanting a percutaneous-tunneled, transfontanellar (PTT) external ventricular drain (EVD) via a purely percutaneous technique at bedside, as the first measure in a stepwise approach (Fig. 1).

\section{Surgical Technique}

All procedures were performed by the pediatric neurosurgeon in 3 different NICUs located in the same regional area of Italy (Emilia-Romagna; 4,500,000 inhabitants) and under strict aseptic conditions; antibiotic prophylaxis with vancomycin and piperacillin/tazobactam was administered perioperatively. The patient, under general anesthesia, was supine with the head slightly elevated. The entry site for the ventricular drain (right lateral edge of the bregmatic fontanel) and the catheter's exit site (temporal region) were marked, and the surgical field was draped.

The entry site was punctured with an intravenous cath-

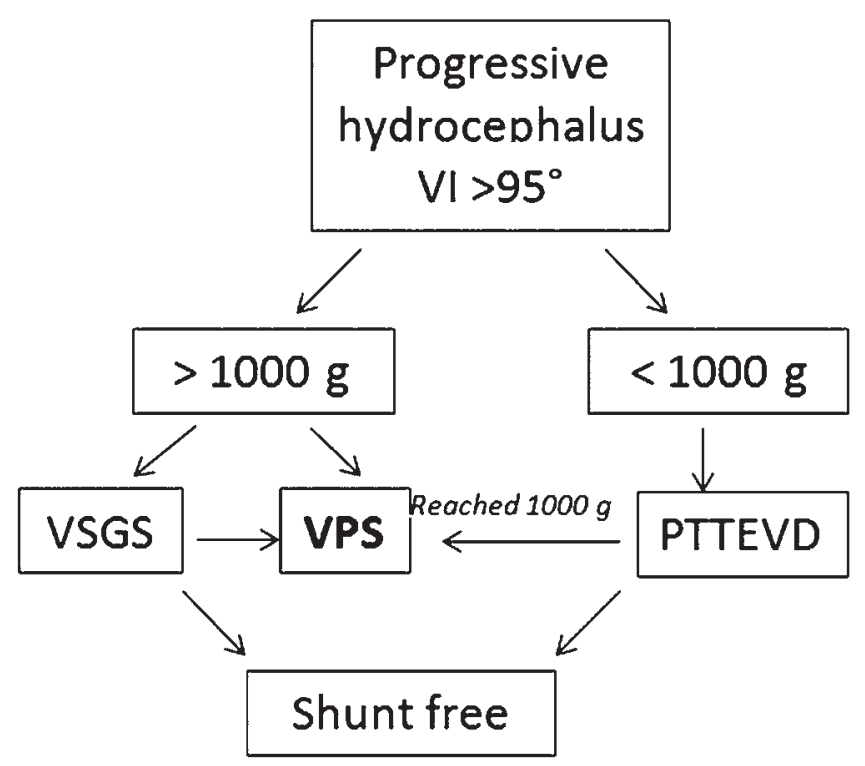

FIG. 1. Stepwise approach to PHH with the introduction of a PTTEVD in ELBW infants. VI = ventricular index; VPS = ventriculoperitoneal shunt; VSGS = ventriculosubgaleal shunt.

eter (14-gauge, $2.1 \times 45 \mathrm{~mm}$, BD Insyte) and subcutaneously tunneled from the fontanel edge to the temporal exit site, leaving the sleeve in place. A blunt, multiple side-hole catheter made of polyvinyl chloride (4-Fr, $2 \times 400 \mathrm{~mm}$, Unomedical-Convatec) was threaded into the sleeve distally to proximally (Fig. 2A); then the sleeve was removed, leaving the drain inside the subcutaneous tunnel.

With the needle tip, a small perforation of the dura mater (smaller than the catheter diameter) was made at the entry site. Then the smoothed distal extremity of the drain was gently inserted through the dura and fed into the ventricle under ultrasonographic control (ipsilateral mastoid view; Fig. 3). Tight adhesion of the catheter to the dural ring was achieved to minimize the risk of CSF leakage. Once the drain was properly placed, passive dripping of CSF was observed from its external end. Afterward, the external end was slid outward through the subcutaneous tunnel, and the drain was fixed with Steri-Strips at the exit site, while the entry site and tunnel were sealed with fibrin glue and covered with soft sterile gauze (Fig. 2B-D). To allow repeated CFS tapping, the external end of the PTTEVD was closed with a sterile plug device and protected with sterile bandages.

\section{Results}

The characteristics of the patients are listed in Table 1. The mean patient weight was $750 \mathrm{~g}$, the average duration of the procedure was 7 minutes, and there was no blood loss.

In all cases but 2, there was no history of sepsis. In Case 2 , the PTTEVD was implanted in a patient who weighed more than the other patients, obtaining resolution of the infection and treating the hydrocephalus in the same way as with a traditional EVD, while maintaining the advantages of a quick, minimally invasive, bedside procedure. 

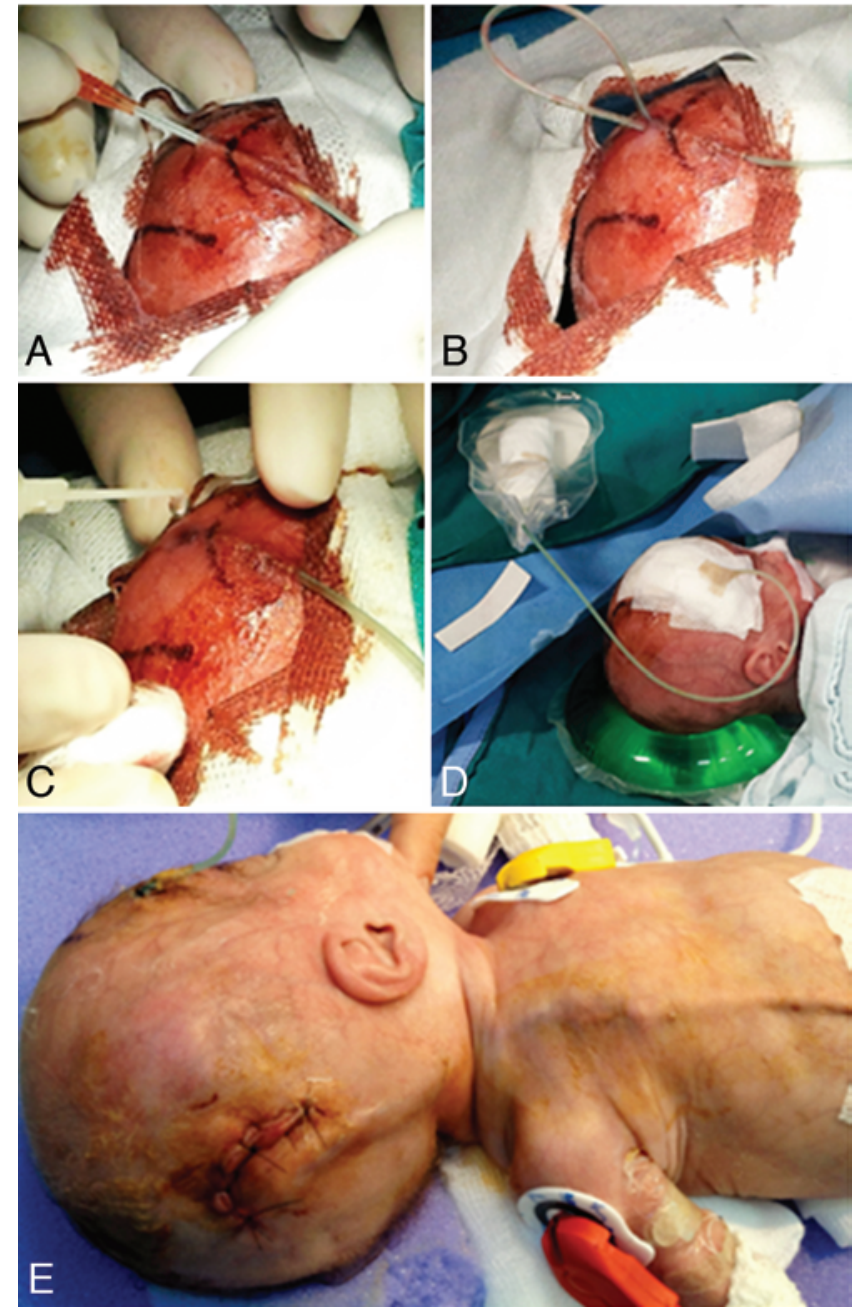

FIG. 2. Positioning of the PTTEVD (A-D). After removal of the PTTEVD and shunt implantation $(E)$. Figure is available in color online only.

The patient in Case 5 died of Streptococcus sepsis, a systemic infection existing prior to PTTEVD placement and remaining unresolved (sterile CSF samples).

After PTTEVD placement, twice-daily removal of CSF (approximately $5 \mathrm{ml}$ until the fontanellar tension became normal) was started and performed by the neonatologist. Additionally, brain ultrasonography scans were obtained by placing properly sterilized devices on the probe and were evaluated daily, and in cases of ventricular enlargement, the amount of CSF removal was adjusted. Each CSF sample was submitted to bacterial culture and cytochemical evaluation. Antibiotics were administered during the entire drainage period. Whenever possible, efforts to maintain asepsis in the daily management of the child were made.

The PTTEVD remained in place for an average of 24 days; no infection was detected on serial CSF cultures except in 1 case in which, on the day after abdominal surgery with ileostomy, an Enterococcus was detected in the CSF sample. Consequently, the patient was immediately started on a 7-day cycle of daily intrathecal vancomycin ( $2 \mathrm{mg}$ at $1 \mathrm{mg} / \mathrm{ml}$ concentration, taking into account the

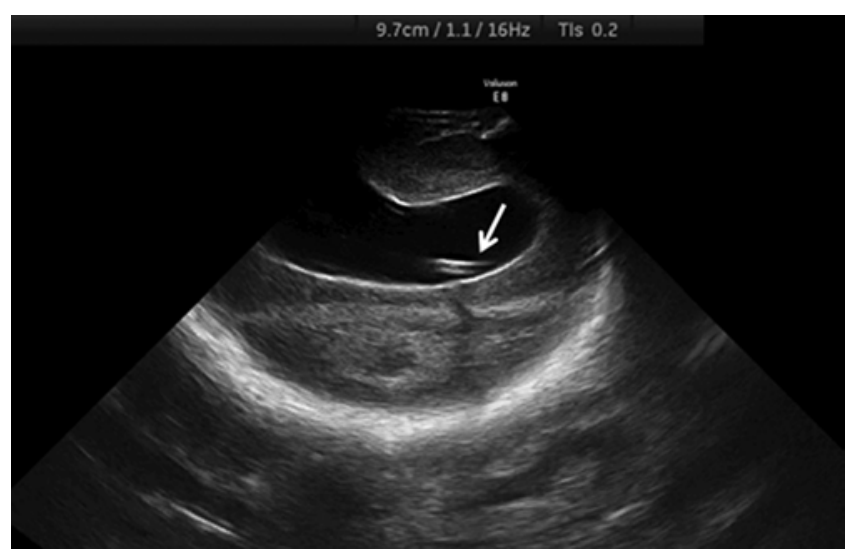

FIG. 3. Ipsilateral mastoid ultrasonography view during PTTEVD (arrow) placement.

extra infusion volume of the PTTEVD). Bacteria in the CSF were eliminated, and the CSF remained sterile for the remaining period of hospitalization.

In the patient in Case 4, a single CSF leakage episode occurred as a result of insufficient CSF withdrawal: oncedaily removal of CSF was scheduled, CSF leakage occurred, and after resuming twice-daily CSF removal no more leaks were observed.

Once a patient reached $1 \mathrm{~kg}$ of weight, when necessary (progressive ventricular dilation after suspension of CSF withdrawal), a ventriculoperitoneal shunt (occipital entry site) was implanted and the PTTEVD was removed.

According to available data on the patients receiving shunts, the follow-up period ranged from 7 to 34 months. In 1 case, after 8 months, the shunt was revised because of intracranial catheter occlusion, but no shunt infection was observed.

\section{Discussion}

The treatment of PHH in ELWB infants still represents a challenge for the pediatric neurosurgeon, particularly when the patient weighs far less than $1000 \mathrm{~g}$. In these cases, the advantages of early treatment in terms of neurological outcome do not always outweigh the surgical risks, especially considering the great difference in the surgical risk before patient weight increases. In fact, before the introduction of our technique, our experience in managing such extreme cases led us to try to postpone treatment, not only because of the risks of CSF leakage, skin dehiscence, infection, and overdrainage, but also because of the global and anesthesiology risks related to surgery on delicate and hemodynamically unstable patients.

The optimal timing of intervention has not yet been established; thus, we decided to place the PTTEVD when the ventricular indexes were significantly above the 95th percentile reference intervals ${ }^{4,5,14}$ without waiting for the appearance of signs and symptoms of raised intracranial pressure ${ }^{6}$ In all cases, to initiate neurosurgical treatment, in addition to the progressive ultrasonography findings (ventricular index $>95$ th percentile), evidence of progressive growth of the head circumference was considered necessary; diastasis of the cranial sutures, dilated scalp veins, 


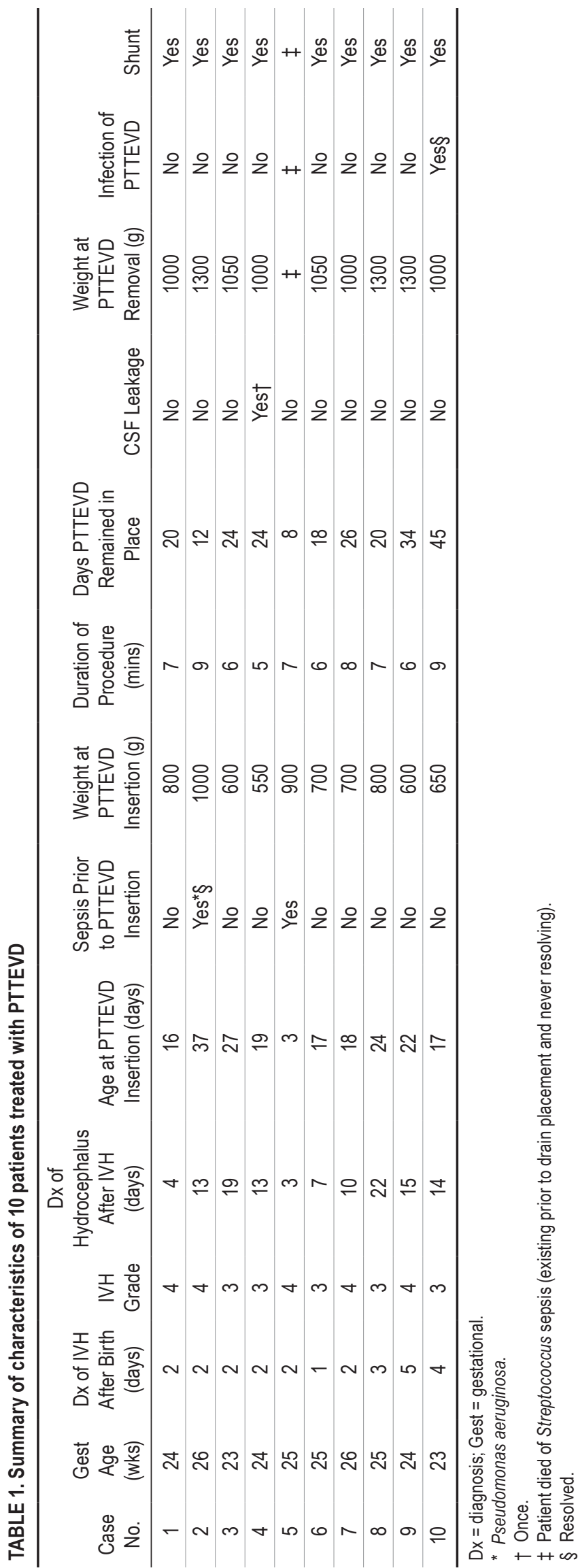

and bulging of the anterior fontanel have never occurred. As a matter of fact, the early treatment of hydrocephalus in preterm infants with $\mathrm{PHH}$ and no initial parenchymal injuries is associated with reduced rates of cognitive, communicative, and social disabilities, although in our series motor function and cerebral palsy rates had not shown any modification..$^{3,6}$ Thus, our stepwise approach might allow surgeons to accelerate the timing of intervention even in babies with temporary contraindications to neurosurgical approaches.

Although the placement of a permanent CSF drain represents the definitive therapeutic option for $\mathrm{PHH}$, it should not be considered a first-line treatment in ELBW infants given its high risks of obstruction, infection, and skin ulceration. ${ }^{18,19}$ Thus, alternative protocols are needed to manage the progressive ventricular dilation while waiting for improved medical conditions before placing a permanent CSF drain.

Positioning a subcutaneous ventricular reservoir is considered a safe and effective procedure in preterm infants; ${ }^{5}$ however, the associated risks in ELBW infants (for example, skin ulceration over the reservoir area) are not negligible and should be taken into account.

As an alternative, positioning a ventriculosubgaleal shunt represents a valid therapeutic option for PHH in infants, and many series have reported the routine use of this option even in ELBW infants, with a variable occurrence of complications..$^{10,13,17,21}$ However, our experience with this technique suggests that if a baby weighs less than $1 \mathrm{~kg}$, the thinness of the skin can become an issue, especially if the consequent subgaleal CSF buildup increases significantly, with stretching of the tissues and the risk of CSF leakage. Moreover, the implantation of a ventriculosubgaleal shunt requires a longer surgical time and a wide skin incision, as compared with those needed in the pure percutaneous technique of PTTEVD placement (with an average 7-minute procedure duration and no blood loss).

Serial lumbar punctures are often performed when the hydrocephalus is communicating. Despite its simplicity, this procedure is associated with increased CSF infections with no evidence of improvement in the rate of shunt placement, disability, and death. ${ }^{20}$

Therefore, the implantation of an EVD may represent a suitable and feasible choice for the stepwise management of PHH in ELBW infants with unstable medical conditions. However, even the simple implantation of a standard EVD in such small infants can cause some issues. For example, the dimensions of available catheters do not fit with the dimensions of the infant head. The standard surgical step needed for EVD positioning includes a surgical wound, skull drilling, and a suture, which are risky and dangerously time-consuming for an unstable and delicate patient. Finally, the standard connection with a standard external collection system may lead, in cases of overdrainage, to the risk of brain collapse and intracranial hemorrhage. In cases of underdrainage, there might be CSF leakage due to excessive CSF pressure, which is released through the wound.

Percutaneous-tunneled, transfontanellar external ventricular drainage represents an adaptation of the standard drainage technique intended to minimize the risks for pa- 
tients: the drain dimensions are adequate to the infant's size, the transfontanellar access makes bone drilling unnecessary, the subcutaneous tunneling and fibrin glue reduce the risk of CSF leakage, and the purely percutaneous technique of implantation allows us to avoid wounds and minimize the duration of surgery. The serial CSF removals produce the same effect as the repeated reservoir tapping without the risks associated with surgical reservoir implantation and the skin damage caused by the repeated transcutaneous reservoir tapping. Another advantage of the small tube dimension is the very low flow rate: the spontaneous CSF withdrawal is slow, gentle, steady, and easily controllable, thus preventing rapid intracranial volume modification. Moreover, the baby can move during CSF removal, and no immobilization is needed. Finally, in comparison with traditional external ventricular drainage, the absence of the CSF line and the external collection system makes nursing the baby in the incubator easier and the ICU setting less demanding for the staff.

Interestingly, the possible catheter obstruction that we expected (as the catheter we used was much thinner than traditional ones), which is especially a factor in the presence of severe IVH, did not occur, and in no case was it necessary to flush the device. In all cases the PTTEVD remained patent for the whole duration of the implantation. We think that the ultrasonography-guided positioning of the catheter (Fig. 3) far from the bigger clot may have contributed to this result.

Another advantage is, in the case of infection, the possibility of immediately starting intrathecal antibiotic therapy, and if no improvement is observed, the PTTEVD can simply be pulled out at bedside (not feasible without another surgery if a previously implanted device such as a reservoir or ventriculosubgaleal shunt is present) simultaneously with the contralateral implantation of another PTTEVD.

The high number of shunting procedures required in the present series could be attributable to the decision to not wait for greater weight gain (which would have otherwise resulted in a greater percentage of shunt-free patients) to reduce the risk of infection. As a matter of fact, if early removal of the catheter is associated with a reduced infection rate, early shunting can be associated with a higher complication rate..$^{2,3,8,15,21}$ Placing a shunt at a patient weight of $1 \mathrm{~kg}$, in our experience, represents an acceptable compromise between the surgical risks and the likelihood of infection with a long-standing PTTEVD. On the other hand, there is probably a greater need for shunts (which will be removed in the future, if possible, by performing a secondary third ventriculostomy). Finally, considering the limited number of studies available on external ventricular drainage in ELBW infants, we believe that future investigations should be aimed at clarifying whether the prolonged use of a PTTEVD is actually related to a higher infection rate and a reduced need for shunting.

\section{Conclusions}

When performed in ELBW infants, standard therapeutic procedures for $\mathrm{PHH}$ are burdened with high risks that do not easily allow early intervention. The introduction of PTTEVD placement to our standard PHH treatment protocol produced a safe and early bedside option for this delicate subgroup of patients. Although the limited sample size does not allow definitive statements, the features of minimal skin traumatisms, absent blood loss, very short operation times, reversibility at bedside, few and manageable infectious complications, and no CSF leakage make this procedure a valid option in the multistep treatment of $\mathrm{PHH}$ in ELBW infants.

\section{Acknowledgments}

A special thanks to Dr. Carmelo Sturiale for the concrete support in all the phases of the present study.

\section{References}

1. Adams-Chapman I, Hansen NI, Stoll BJ, Higgins R: Neurodevelopmental outcome of extremely low birth weight infants with posthemorrhagic hydrocephalus requiring shunt insertion. Pediatrics 121:e1167-e1177, 2008

2. Arabi Y, Memish ZA, Balkhy HH, Francis C, Ferayan A, Al Shimemeri A, et al: Ventriculostomy-associated infections: incidence and risk factors. Am J Infect Control 33:137-143, 2005

3. Bassan H, Eshel R, Golan I, Kohelet D, Ben Sira L, Mandel D, et al: Timing of external ventricular drainage and neurodevelopmental outcome in preterm infants with posthemorrhagic hydrocephalus. Eur J Paediatr Neurol 16:662-670, 2012

4. Brouwer MJ, de Vries LS, Groenendaal F, Koopman C, Pistorius LR, Mulder EJ, et al: New reference values for the neonatal cerebral ventricles. Radiology 262:224-233, 2012

5. Brouwer AJ, Groenendaal F, van den Hoogen A, VerboonMaciolek M, Hanlo P, Rademaker KJ, et al: Incidence of infections of ventricular reservoirs in the treatment of posthaemorrhagic ventricular dilatation: a retrospective study (1992-2003). Arch Dis Child Fetal Neonatal Ed 92:F41$\mathrm{F} 43,2007$

6. Brouwer A, Groenendaal F, van Haastert IL, Rademaker K, Hanlo P, de Vries L: Neurodevelopmental outcome of preterm infants with severe intraventricular hemorrhage and therapy for post-hemorrhagic ventricular dilatation. J Pediatr 152:648-654, 2008

7. Cinalli G: Alternatives to shunting. Childs Nerv Syst 15:718-731, 1999

8. Collins CD, Hartley JC, Chakraborty A, Thompson DN: Long subcutaneous tunnelling reduces infection rates in paediatric external ventricular drains. Childs Nerv Syst 30:1671-1678, 2014

9. Januschek E, Machado LS, Steinthal B, Ulrich PT: Posthemorrhagic hydrocephalus in very low birth weight infants - a new gentle surgical technique for external ventricular drainage. Childs Nerv Syst 27:991-994, 2011

10. Fulmer BB, Grabb PA, Oakes WJ, Mapstone TB: Neonatal ventriculosubgaleal shunts. Neurosurgery 47:80-84, 2000

11. Futagi Y, Suzuki Y, Toribe Y, Nakano H, Morimoto $\mathrm{K}$ : Neurodevelopmental outcome in children with posthemorrhagic hydrocephalus. Pediatr Neurol 33:26-32, 2005

12. Jian L, Hang-song S, Zheng-lang L, Li-sheng Y, Heng W, $\mathrm{Nu} \mathrm{Z}$ : Implantation of Ommaya reservoir in extremely low weight premature infants with posthemorrhagic hydrocephalus: a cautious option. Childs Nerv Syst 28:1687-1691, 2012

13. Kariyattil R, Mariswamappa K, Panikar D: Ventriculosubgaleal shunts in the management of infective hydrocephalus. Childs Nerv Syst 24:1033-1035, 2008 
14. Levene MI: Measurement of the growth of the lateral ventricles in preterm infants with real-time ultrasound. Arch Dis Child 56:900-904, 1981

15. Mayhall CG, Archer NH, Lamb VA, Spadora AC, Baggett JW, Ward JD, et al: Ventriculostomy-related infections. A prospective epidemiologic study. N Engl J Med 310:553559,1984

16. McCrea HJ, Ment LR: The diagnosis, management, and postnatal prevention of intraventricular hemorrhage in the preterm neonate. Clin Perinatol 35:777-792, vii, 2008

17. Tubbs RS, Smyth MD, Wellons JC III, Blount J, Grabb PA, Oakes WJ: Life expectancy of ventriculosubgaleal shunt revisions. Pediatr Neurosurg 38:244-246, 2003

18. Whitelaw A, Aquilina K: Management of posthaemorrhagic ventricular dilatation. Arch Dis Child Fetal Neonatal Ed 97:F229-F233, 2012

19. Whitelaw A, Thoresen M, Pople I: Posthaemorrhagic ventricular dilatation. Arch Dis Child Fetal Neonatal Ed 86:F72-F74, 2002

20. Whitelaw A: Repeated lumbar or ventricular punctures in newborns with intraventricular hemorrhage. Cochrane Database Syst Rev (1):CD000216, 2001

21. Willis BK, Kumar CR, Wylen EL, Nanda A: Ventriculosubgaleal shunts for posthemorrhagic hydrocephalus in premature infants. Pediatr Neurosurg 41:178-185, 2005

\section{Disclosures}

The authors report no conflict of interest concerning the materials or methods used in this study or the findings specified in this paper.

\section{Author Contributions}

Conception and design: Zucchelli. Acquisition of data: Zucchelli, Lefosse, Corvaglia, Martini, Sandri, Soffritti, Ancora, Mammoliti, Gargano. Analysis and interpretation of data: Zucchelli. Drafting the article: Zucchelli. Reviewed submitted version of manuscript: Zucchelli, Galassi. Approved the final version of the manuscript on behalf of all authors: Zucchelli. Study supervision: Zucchelli.

\section{Correspondence}

Mino Zucchelli, Pediatric Neurosurgery, IRCCS Istituto delle Scienze Neurologiche di Bologna, via Altura 3, Bologna 40139, Italy.email:minoz@inwind.it. 